\title{
Effect of Cognitive Load on Postural Stability
}

\author{
Kristyna Rusnakova ${ }^{1 *}$, David Gerych ${ }^{1,2}$ and Miloslav Stehlik ${ }^{1}$ \\ ${ }^{1}$ CASRI, Czech Republic \\ ${ }^{2}$ Department of Military Internal Medicine and Military Hygiene, Faculty of Military Health Sciences in Hradec Kralove, \\ University of Defence in Brno, Czech Republic
}

\begin{abstract}
Postural stability is the dynamics of the postural control system associated with maintaining balance during quiet standing. Postural control during normal upright stance in humans is a well-learned task. The control of posture is affected by physiological factors such as the muscular and the skeletal system, fatigue, or psychological factors such as the anxiety arousal, state of mind, attention and cognitive load. This study investigates the effects of cognitive load on postural stability in airborne units of czech army. Postural sway using static posturography was assessed in 18 pilots before and after cognitive load. Postural stability was quantified using force plate measures of center of pressure (COP) and center of gravity (COG), cognitive load was assessed by selected tests for spatial abilities while obstructing listening loud sound (crying baby). Each standing trial was performed with eyes open and eyes close for $60 \mathrm{~s}$. Results showed that cognitive load led to less body sway, while individuals had a smaller ellipse area of center of gravity after cognitive load compared with a default situation. In conclusion, control of body sway and cognitive functioning are to some extent related, cognitive load can arouse concentration and can affect postural stability.
\end{abstract}

KEYWORDS: Airborne; Cognitive load; Postural stability; Spatial abilities

\section{INTRODUCTION}

The ability to maintain postural stability is the foundation of achieving independent standing and walking [1]. It is a result of an integration of vestibular, somatosensory, and visual systems to maintain the center of mass within the base of support [24]. Any deficit in these components will result in poor posture control, which is often associated with the risk of falling and has been identified as a major health problem [5]. Many physical and psychological factors factors can affect postural sway [6]. With a focus on psychological factors, we consider the impact of a state of anxiety, a state of mind, an attention, a cognitive task etc. Human postural stability might be negatively influenced by muscle fatigue [7-9], but it is still unclear whether or how is postural control affected by the cognitive fatigue.
Numerous studies investigated association between the postural control and the cognitive function using dual task designs in which subjects have performed cognitive and postural tasks simultaneously [10]. Cognitive task affects the characteristics of the neuromuscular response that underlie the control of reactive balance. The muscle activity decline while performing the secondary task that suggests that less attentional processing capacity is available for balance control during the dual-task paradigm. The decline of muscle activity when the secondary task is performed suggests that less attentional processing capacity is available for balance control during the dual-task paradigm. The dual-task activity has a greater impact on balance control in the older adults than in the young adults [11]. These studies indicate that the postural control and attentional resources are related. The

\begin{tabular}{c|l} 
Quick Response Code: & Address for correspondence: Kristyna Rusnakova, CASRI, Czech Republic \\
\cline { 2 - 3 } & Received: April 20,2020 Published: June 17, 2020 \\
& $\begin{array}{l}\text { How to cite this article: Kristyna R, David G, Miloslav S. Effect of Cognitive Load on Postural } \\
\text { Stability. } 2020 \text { - 2(3) OAJBS.ID.000182. DOI: 10.38125/OAJBS.000182 }\end{array}$
\end{tabular}


attention and cognitive load may influence the central processing of information required for the perception and the control of orientation [12]. There is possible causal links between vestibular dysfunction and cognitive performance. Previous literature suggests the inner ear vestibular system has a substantial impact on cognitive function. The strongest evidence exists in connecting vestibular function to the cognitive domain of visuospatial ability, which includes spatial memory, navigation, mental rotation, and mental representation of three-dimensional space. Substantial evidence also exists suggesting the vestibular system has an impact on attention and cognitive processing ability [13]. The static posturography could be a useful diagnostic tool of equilibrium for assessing beginning cognitive decline in men [14].

Not only dual-task exercice but also emotional-based factors such as a state of mind, a state of anxiety or an arousal can affect postural steadiness. The state anxiety, the physiological arousal and the balance efficacy are related to specific changes in postural performance with increased standing balance [15]. According to the theory of arousal, the relationship between activation and power follows the inverted $U$ curve with the optimal degree of activation for each individual task. The more complex the task, the lower the level of activation required for optimum performance [16]. The cognitive load can arouse attentional resources and affect the postural control.

It is still unclear whether or how a cognitive load interacts with postural control during a quiet stance. The purposes of this study were to investigate the effects of cognitive load on changes in postural control induced by a cognitive load.

\section{MATERIALS AND METHODS}

Postural sway was measured in 18 healthy subjects (17 men, 1 woman), aged between 25 and 47 years old. The group consisted of pilots of jet airplanes and pilots of transport and combat helicopters. All of subjects participated voluntarily in this study.

A repeated measures design was employed, in which participants performed two trials of quiet, upright stance before and after cognitive load. Standing trials lasted $60 \mathrm{~s}$, during which participants stood as still as possible on a force platform with fixation a point fixing on the wall or not. Data were collected for $60 \mathrm{~s}$. Therefore, the aim of the present study was to evaluate whether cognitive fatigue alters the postural control in subjects when standing on a stable support surface. Postural control was quantitatively considered by measuring the movement of the center of gravity (COG) and the center of pressure (COP). COP is defined as the center of distributed total force applied to a supporting surface. COG is the mean location of gravitational force acting on the body
[17]. The cognitive load was represented by cognitive tests of spatial abilities while listening to a highly stressful recording of a baby crying. The loud sound of a baby crying interferes with primary task performance and makes performing a task more difficult. Baby crying provokes an emotional response that subconsciously affects behavior. That is the reason why it is so difficult to ignore the sound of a crying baby [18]. For the assessment of spatial abilities, Test No. 07 - selection of shapes - and Test No. 08 - tasks with cubes from the Intelligence Structure Test 2000 R (I-S-T 2000 R) - were used [19]. Test No. 07 included twenty items. Each item showed a single pattern cut into several pieces. Participants were asked to find out which of the five patterns can be obtained by organizing individual pieces so that nothing is missing. Test No. 08 comprised twenty items and was based on mental abilities that require the spatial transformation of a perceived object. Five cubes in changed positions had to be entered, with only three sides visible at a time. One of the five cubes had to be matched to the one with the right rotation. Participants were given seven minutes to complete both tests. The participants were taught how to complete the cognitive tests before the time began to be measured and were given headphones with a loud baby crying record. In short, the potential effect of background listening for a cognitive test is that it requires someone's attention. A Nintendo Wii Balance Board was used for measurement of the distance (D), the mean velocity (MV) and the ellipse area (EA) of subjects under the eyes open and eyes closed conditions in a standing posture. The Nintendo Wii Balance Board is a dual-plate force plate, instrumented with four uni-axial stain gauge transducers positioned in each corner which are used to assess force distribution and the resultant movements in COP. Data were transferred wirelessly to a personal computer via Bluetooth. COP was calculated separately for left (L COP) and right leg (R COP). The effect of cognitive load on postural stability was analysed using the Wilcoxon matched-pairs sign rank test; each parameter being evaluated separately. All statistical calculations were done with the "Statistica 13" statistical software.

Commonly used measures of COP and COG in the time and frequency domains (D, MV, EA) were used. EA covered by the trajectory of the COP with a $90 \%$ confidence interval, where the smaller the surface is, the better the performance is. MV represents the total distance covered by COP divided by the duration of the sampled period and constitutes a good index of the amount of activity required to maintain stability [20]. D is the total length travelled by the COP to reach the maximal distance - the smaller the distance, the better the postural stability [21]. The reliability and validity of these parameters are better reported for $95 \%$ confidence ellipse area of COP and sway velocity than other COP parameters [22-23].

\section{RESULTS}

Table 1: Summary of Wilcoxon Matched Pairs Test results (P values) for the effects of cognitive load on COG.

\begin{tabular}{|c|c|c|c|c|}
\hline \multirow[t]{2}{*}{ Pair of Variables } & \multicolumn{4}{|c|}{$\begin{array}{c}\text { Wilcoxon Matched Pairs Test (BEFORE_AFTER_COG) Marked } \\
\text { Tests are Significant at } p<0.05000\end{array}$} \\
\hline & Valid $\mathbf{N}$ & $\mathbf{T}$ & $\mathbf{Z}$ & $p$-value \\
\hline Distance_eyes_open \& Distance_eyes_open & 18 & 56 & 1.284735 & 0.198886 \\
\hline MeanVel_eyes_open \& MeanVel_eyes_open & 18 & 56 & 1.284735 & 0.198886 \\
\hline EllipseArea_eyes_open \& EllipseArea_eyes_open & 18 & 31 & 2.373494 & 0.017621 \\
\hline Distance_eyes_close \& Distance_eyes_close & 18 & 80 & 0.239527 & 0.810697 \\
\hline MeanVel_eyes_close \& MeanVel_eyes_close & 18 & 80 & 0.239527 & 0.810697 \\
\hline EllipseArea_eyes_close \& Ellipse Area_eyes_close & 18 & 33 & 2.286394 & 0.022232 \\
\hline
\end{tabular}


Table 2: Summary of Wilcoxon Matched Pairs Test Results (P values) for the effects of cognitive load on LCOP.

\begin{tabular}{|c|c|c|c|c|}
\hline \multirow{2}{*}{ Pair of Variables } & \multicolumn{3}{|c|}{ Wilcoxon Matched Pairs Test (BEFORE_AFTER_COG) Marked tests are } \\
significant at p < 0.05000
\end{tabular}

Table 3: Summary of Wilcoxon Matched Pairs Test results ( $P$ values) for the effects of cognitive load on RCOP.

\begin{tabular}{|c|c|c|c|c|}
\hline \multirow{2}{*}{ Pair of Variables } & \multicolumn{4}{|c|}{ Wilcoxon Matched Pairs Test (BEFORE_AFTER_COG) Marked tests are } \\
& & significant at p $<0.05000$ & Z & p-value \\
\cline { 2 - 5 } & Valid N & 72 & 0.58793 & 0.55658 \\
\hline Distance_eyes_open \& Distance_eyes_open & 18 & 72 & 0.58793 & 0.55658 \\
\hline MeanVel_eyes_open \& MeanVel_eyes_open & 18 & 72 & 0.58793 & 0.55658 \\
\hline EllipseArea_eyes_open \& EllipseArea_eyes_open & 18 & 74 & 0.500829 & 0.616492 \\
\hline Distance_eyes_close \& Distance_eyes_close & 18 & 74 & 0.500829 & 0.616492 \\
\hline MeanVel_eyes_close \& MeanVel_eyes_close & 18 & 50 & 1.546038 & 0.122096 \\
\hline EllipseArea_eyes_close \& EllipseArea_eyes_close & 18 & & 74 \\
\hline
\end{tabular}

Acute effect of cognitive load was observed across all dependant variables in an open eye measurement. Significant effect of cognitive load was found among parameters of COG and COP, specifically in ellipse area. In a trial with eyes open, CL caused a significant difference $(\mathrm{p}<0.05)$ in EA of COG (decreased by $25 \%$ ), in D of L COP (decreased by $28 \%$ ) and in MV of L COP (decreased by $28 \%$ ). In a close-eye experiment, EA of COG also significantly fell (decreased by 29 \%) (See Chyba! Nenalezen zdroj odkazů., Chyba! Nenalezen zdroj odkazů., (Table 1-3) for a summary of Wilcoxon Pairs Test results with statistically significant variables).
Data concerning differences in postural sway are summarized in boxplots (Figure 1-4). The most substantial effects of COG were evident in a trial with eyes close with a $29 \%$ decrease in EA of COG. In contrast, no significant increasing of COG and COP were found across all measurements. Decreases in variables of COP and COG to be tended to improve postural stability while subject's postural characteristics improved after cognitive load compared with a default situation. The cognitive load had a positive effect on postural stability.

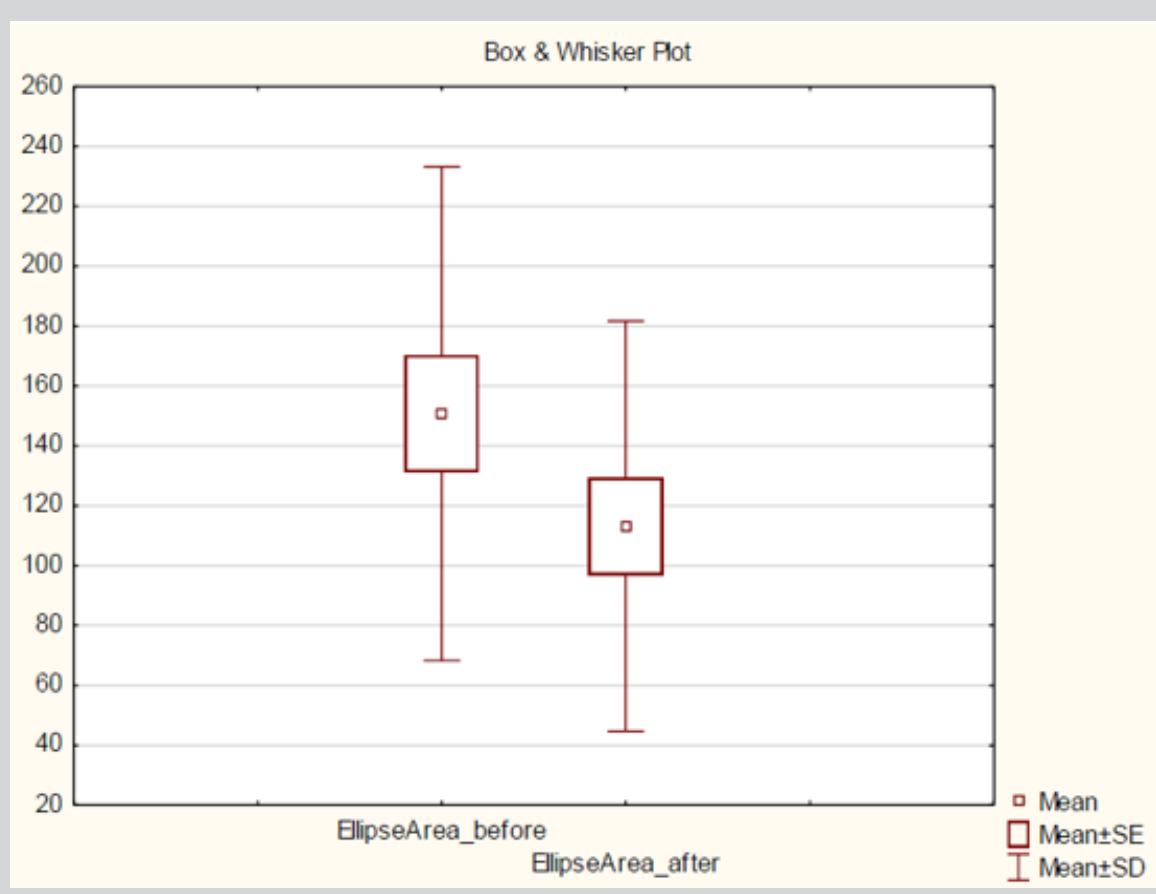

Figure 1: Mean values of Ellipse Area of COG before and after cognitive load in a open-eyes trial. 


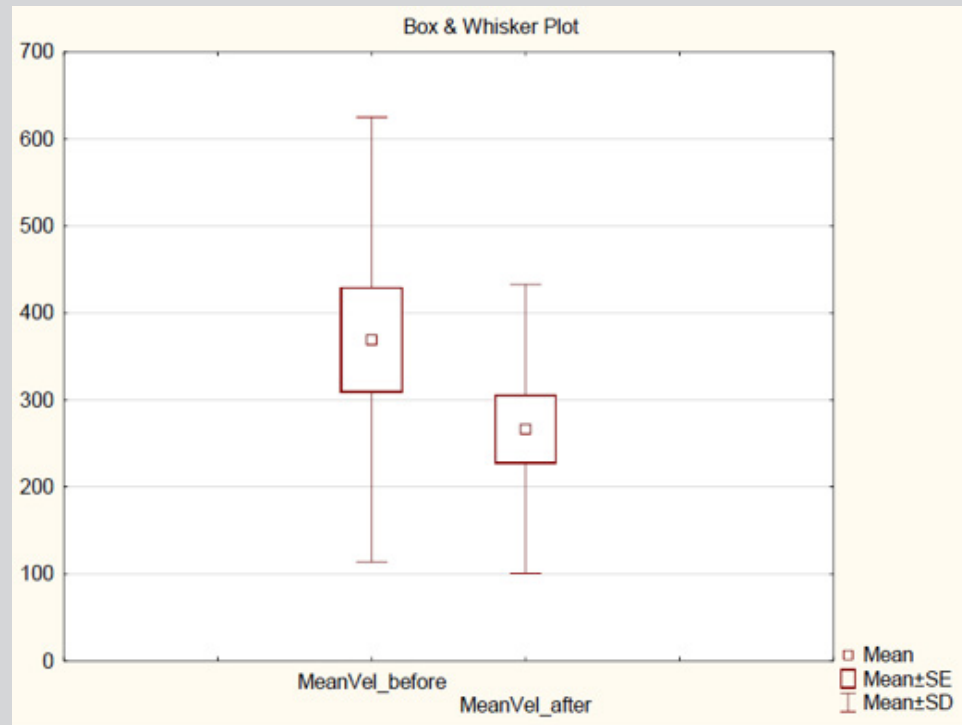

Figure 2: Mean values of Velocity of LCOP before and after cognitive load in a open- eyes trial.

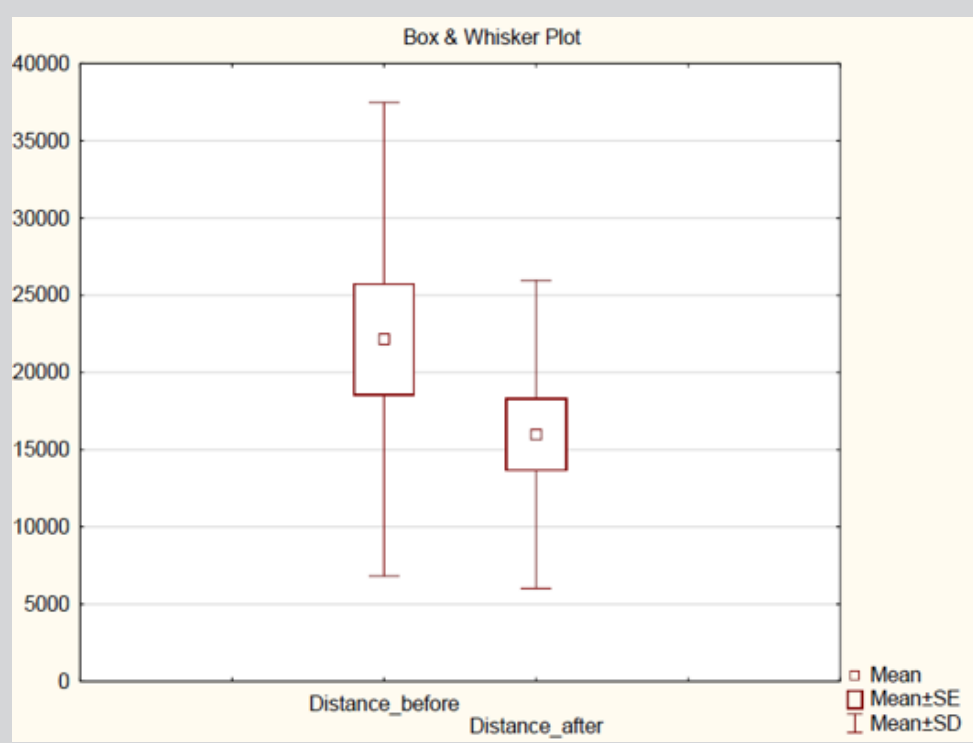

Figure 3: Mean values of Distance of LCOP before and after cognitive load in a open-eyes trial.

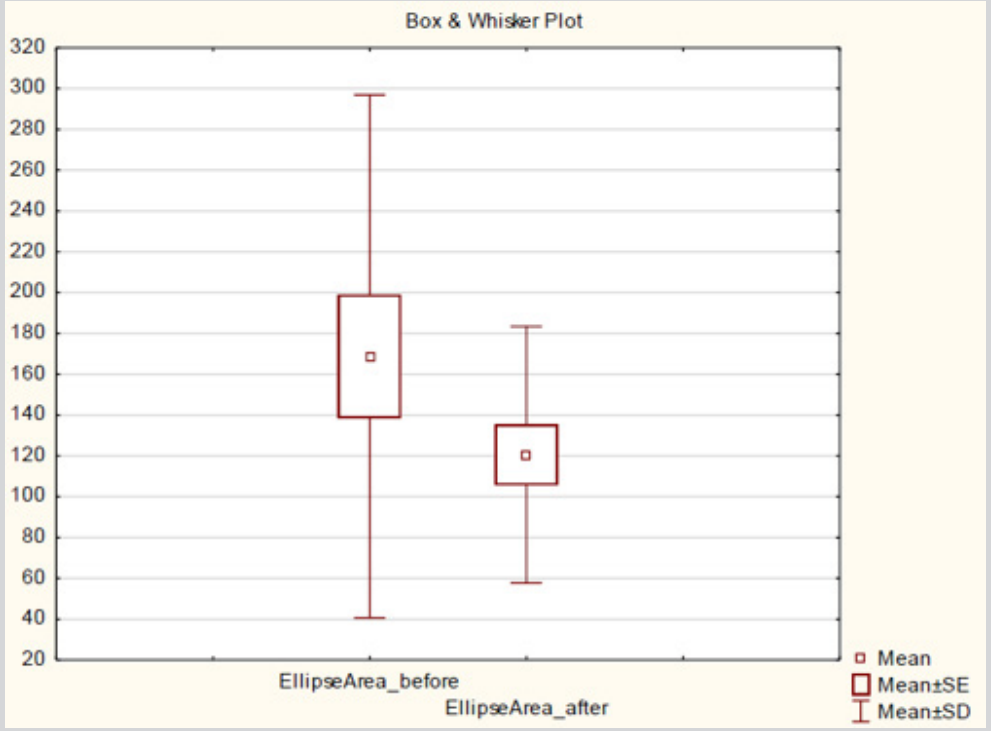

Figure 4: Mean values of Ellipse Area of COG before and after cognitive load in a close-eyes trial. 


\section{DISCUSSION}

The purposes of this study were to investigate the effects of cognitive load induced by cognitive tests while listening a recording of a crying baby on changes in postural control. A repeated measures design was employed, in which participants performed multiple trials of quiet, upright stance before and after cognitive load including tests of spatial abilities while listening a highly stressful recording of a crying baby. The postural sway was assessed using force platforms, participants being in static position with eyes open and eyes closed. Standing trials lasted $60 \mathrm{~s}$. Main effect of cognitive load was observed at EA of COG (in both trials), in D of L COP (in a trial with eyes open) and in MV of L COP (in a trial with eyes open) consistent with hypothesized changes in postural control after cognitive load. The results of this study demonstrated that control of posture is influenced by the cognitive load while the cognitive load may arouse attentional resources and have a positive effect on individual's postural stability. The generalizability of the current results is limited by some issues. The sample size is relatively small, while more accurately results may be gained by involving many participants. Another issue needs to be further investigated by considering cognitive and emotional factors separately with the emphasis of assessment the relationship between vestibular system and cognitive function. Results of this study may facilitate the development of strategies to improve acute postural stability of pilots.

\section{CONCLUSION}

We assert that cognitive load induced by spatial skills tests and disruptive recording may improve performance in postural stability during quiet standing in airborne units of Czech army. As previously mentioned, postural stability is a complex task that requires coordination of visual, vestibular, and somatosensory inputs, and numerous investigations have been performed to elucidate varied factors affecting the maintenance of stability. In this study, various parameters that quantify postural stability have been reviewed. Cognitive load led to improved postural stability performance, but it is still not clear which specific aspects of cognitive stress caused it. These results suggest that there is a possible relationship between the performance and the arousal. Increased arousal can help improve performance but only up to a certain point. At the point when arousal becomes excessive, performance diminishes. Load induced cognitive tests and emotional stress was probably not large enough to lead to a reduction in performance in postural stability. Soldiers are trained to deal with stressful situations during training involving extreme endurance training, sleep deficit and psychic stress. Their mental and physical resilience is therefore at a very high level; it may be difficult to evoke an appropriate psychological stress. We can also consider connections between cognitive functions, especially spatial abilities, and postural stability. Further research is needed to identify what aspects of cognitive load caused improvements in postural stability parameters.

\section{REFERENCES}

1. Melzer I, Benjuya N, Kaplanski J (2004) Postural stability in the elderly: a comparison between fallers and non-fallers. Age Ageing 33(6): 602607.

2. Alcock L, O’Brien TD, Vanicek N (2018) Association between somatosensory, visual and vestibular contributions to postural control, reactive balance capacity and healthy ageing in older women. Health care Women Int 39(12): 1366-1380.
3. Jamison LR, Gines G, Marshall J, Rosario MG (2019) Vestibular and proprioceptive alteration influence postural instability during dual tasks in adults diagnosed with HIV [Texas Woman's University]. Journal of Student Research 9(2).

4. Paniccia M, Wilson KE, Hunt A, Keightley M, Zabjek K, et al. (2018) Postural stability in healthy child and youth athletes: the effect of age, sex and concussion-related factors on performance. Sports Health 10(2): 175-182

5. Winter, David A, Aftab EP, James SF (1990) Assessment of balance control in humans. Med Prog Technol 16(1-2): 31-51.

6. Abbas R, Abadi ZE (2012) The effects of anxiety on balance parameters in young female university students. Iranian J Psychiatry. 7(4): 176-179.

7. Gribble PA, Hertel J (2004) Effect of lower-extremity muscle fatigue on postural control. Arch Phys Med Rehabil 85(4): 589-592.

8. Papa EV, Garg H, Dibble LE (2015) Acute effects of muscle fatigue on anticipatory and reactive postural control in older individuals: a systematic review of the evidence. J Geriatr Phys Ther 38(1): 40-48.

9. Kennedy A, Guevel A, Sveistrup H (2012) Impact of ankle muscle fatigue and recovery on the anticipatory postural adjustments to externally initiated perturbations in dynamic postural control. Exp Brain Res 223(4): 553-562.

10. Yardley L, Gardner M, Leadbetter A, Lavie N (1999) Effect of articulatory and mental tasks on postural control. Neuroreport 10(2): 215-219.

11. Rankin JK, Woollacott MH, Shumway-Cook A, Brown LA (2000) Cognitive influence on postural stability: a neuromuscular analysis in young and older adults. J Gerontol A: Biol Sci Med Sci 55(3): M112-M119.

12. Yardley L, Mark SR (2001) Psychological factors influencing recovery from balance disorders. J Anxiety Disord 15(1-2): 107-119.

13. Bigelow, Robin T, Yuri A (2015) Vestibular involvement in cognition: visuospatial ability, attention, executive function and memory. J Vestib Res 25(2): 73-89.

14. Goto S, Sasaki A, Takahashi I, Mitsuhashi Y, Nakaji S, et al. (2018) Relationship between cognitive function and balance in a communitydwelling population in Japan. Acta otolaryngol 138(5): 471-474.

15. Carpenter MG, Adkin AL, Brawley LR, Frank JS (2006) Postural, physiological and psychological reactions to challenging balance: does age make a difference? Age Ageing 35(3): 298-303.

16. Robert MY, John DD (1908) The relation of strength of stimulus to rapidity of habit-formation. J Comparative Neurol Psychol 18(5): 459482.

17. Jancova J (2008) Measuring the balance control system-review. Acta Medica (Hradec Kralove) 51(3): 129-137.

18. Piallini G, Francesca DP, Alessandra S (2015) Parental brain: cerebral areas activated by infant cries and faces. A comparison between different populations of parents and not. Frontiers in psychology 6: 1625 .

19. Amthauer R, Brocke B, Liepmann D, Beauducel A (2005) Test struktury inteligence IS-T 2000 R. Praha: Testcentrum.

20. Geurts, Alexander CH, Nienhuis B, Mulder TW (1993) Intrasubject variability of selected force-platform parameters in the quantification of postural control. Arch Physical Med Rehabil 74(11): 1144-1150.

21. Lemay JF, Gagnon DH, Nadeau S, Grangeon M, Gauthier C (2014) Centerof-pressure total trajectory length is a complementary measure to maximum excursion to better differentiate multidirectional standing limits of stability between individuals with incomplete spinal cord injury and able-bodied individuals. J Neuroeng Rehabil 11(1): 8.

22. Pinsault N, Vuillerme N (2009) Test-retest reliability of centre of foot pressure measures to assess postural control during unperturbed stance. Medical Eng phy 31(2): 276-286.

23. Bauer C, Gröger I, Rupprecht R, Gaßmann KG (2008) Intrasession reliability of force platform parameters in community-dwelling older adults. Arc Physical Med Rehabil 89(10): 1977-1982. 